\title{
The Mini Astrophysical MeV Background Observatory (MAMBO): A CubeSat for Measuring the MeV Extragalactic Gamma-Ray Background
}

\author{
W. Thomas Vestrand ${ }^{1}$ \\ E-mail:vestrand@lanl.gov
}

Peter Bloser, Andrew Hoover, Lucas Parker \& James Wren

Los Alamos National Laboratory

P.O. Box 1663

Los Alamos, NM 87545 USA

The origin of Extragalactic Background (EGB) light in the $300 \mathrm{keV}-30 \mathrm{MeV}$ energy range is an unsolved question that has persisted for more than 40 years. MAMBO (Mini Astrophysical MeV Background Observatory) is a CubeSat mission concept motivated by the realization that an instrument purpose-built for measuring the MeV EGB from a CubeSat can provide quality measurements that exceed the best that are currently available. There are two key reasons for this: (1) the EGB is so bright at MeV energies that it only requires an instrument with a small effective area and (2) the best existing measurements are compromised by instrumental background, which scales with the mass of the spacecraft/instrument. Here, small is beautiful. We present a design for a gamma-ray spectrometer capable of both providing quality measurements of the spectrum and angular distribution of the MeV EGB and meeting the tight Size, Weight, and Power (SWaP) constraints imposed by deployment on a 6U CubeSat. During a two year baseline mission, MAMBO will be able to collect measurements of the MeV EGB background that are superior to the best currently available and place important constraints on MeV EGB origin.

36th International Cosmic Ray Conference -ICRC2019-

July 24th - August 1st, 2019

Madison, WI, U.S.A. 


\section{Introduction}

An ability to freely traverse cosmic magnetic fields and their long attenuation lengths make gamma-rays important tracers of cosmic ray acceleration in the Universe. Gamma-ray observations in the $\mathrm{MeV}$ energy band can be particularly powerful probes of cosmic ray physics because the continuum emission diagnostics are often augmented by spectral line diagnostics associated with positron annihilation and nuclear de-excitation. Gamma-ray bursts, pulsars, supernovae, active galactic nuclei (AGN) and the Sun are all know to be discrete sources of MeV gamma-ray emission [1,2]. There is also an extended diffuse component of $\mathrm{MeV}$ emission from the galactic plane that is believed to be bremsstrahlung and inverse Compton emission from galactic cosmic ray electrons [3]. But, the least understood source of $\mathrm{MeV}$ gamma-rays is a diffuse component that spans the whole sky and is likely generated at distances beyond the confines of our galaxy [4]. Many of the popular models for the generation of this MeV Extragalactic Background (EGB) light employ cosmic ray interactions in discrete extragalactic sources.

The first observational evidence for the existence of the MeV EGB came in the 1970's from spectrometers deployed on the Apollo 15, 16, and 17 Service Modules during their voyages to the Moon [5]. These early measurements indicated the presence of an isotropic cosmic gamma-ray flux with a broad spectral feature, the so-called "MeV bump", which started near $1 \mathrm{MeV}$ and rolled off near $10 \mathrm{MeV}$ into a steeper power-law at higher energies. By the 1990's, space-based measurements from the Gamma-Ray Spectrometer (GRS) on the Solar Maximum Mission (SMM) and the Compton Telescope (COMPTEL) on the Compton Gamma-ray Observatory (CGRO) showed that the "MeV bump" did not exist and that the MeV EGB flux was fainter than the early Apollo measurements indicated $[6,7,8]$. These more modern spacecraft measurements also showed that the $\mathrm{MeV}$ flux was not consistent with the spectral curvature exhibited by the well characterized x-ray EGB flux and required a power-law extension up to at least $8 \mathrm{MeV}$ (see figure 1). But even today, our knowledge of the properties of the MeV EGB is poor and is well behind our understanding of the $\mathrm{x}$-ray and high-energy $(>100 \mathrm{MeV})$ gamma-ray EGB light. For example, an analysis of observations by SMM GRS was able to provide a $300 \mathrm{keV}-2 \mathrm{MeV}$ spectral measurement of the EGB that had good statistical precision. But since SMM GRS

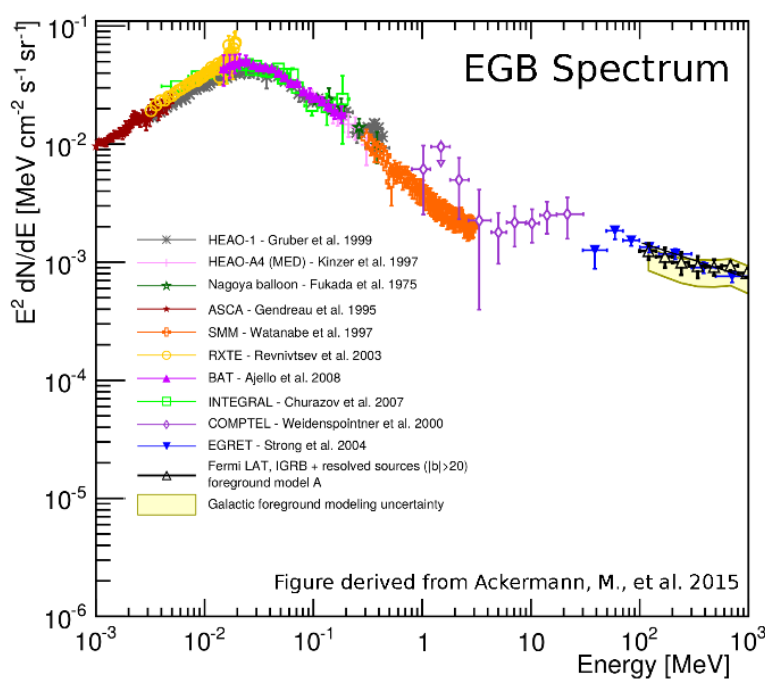

Figure 1. The spectrum of the extragalactic background light from $x$-ray energies to high-energy gamma-ray energies. Adapted from [8]. continuously tracked the Sun, the ecliptic pointing constraint also meant that it could not provide a map of MeV EGB flux at high galactic latitudes. As a result, a full sky map of the MeV EGB is not available and limits on the anisotropy are not well measured [8]. Starting at about $1 \mathrm{MeV}$, COMPTEL was able to provide spectral measurements of the EGB for a few high galactic latitude fields and provide evidence for the emergence of a new spectral component near a few $\mathrm{MeV}$, but the fidelity of the measurements are limited by large systematic errors associated with instrumental background contamination [6]. 
There are many intriguing ideas and models for the generation of the MeV EGB light. Potential source candidates range from Dark Matter annihilation [10], to gamma-rays from the SN Ia Supernovae that are used to trace the effects of Dark Energy [11], to emission powered by massive black holes in Blazars [12], to magnetic reconnection in the coronae of Seyfert galaxies [13] or intense cosmic ray fluxes in star forming galaxies[14]. The roadblock to determining the source(s) and understanding the origin of the MeV EGB light is the poor quality and incompleteness of the existing observations. To resolve the MeV EGB origin puzzle, as was pointed out in a recent Astro2020 Science White Paper [4], one needs a full sky map of the MeV EGB spectral shape and intensity collected with a low instrumental background spectrometer.

\section{The Observational Challenge}

The fact that astrophysical gamma-ray sources are faint means that space-based gamma-ray observatories employ instruments with large collecting areas and a spacecraft bus that is as large as possible. But radiation belt particles, solar energetic particles and galactic cosmic rays striking these massive structures generate an intense background of secondary products (gamma-rays and neutrons) and radioactivity - a background "fog"- that compromise the ability of traditional gamma-ray missions to measure the EGB at MeV gamma-ray energies. The intensity of this instrumental background depends on the details of the observatory orbit (e.g. low earth orbits that do not traverse the South Atlantic Anomaly are better than those that do [15]). But, for any given orbit, the intensity of this contaminating instrumental background scales roughly with the overall mass of the observatory. It is the control of the instrumental systematic errors associated with this locally generated background that poses the greatest observational challenge for MeV EGB measurements.

\section{The Micro Astrophysical $M e V$ Background $O$ bservatory (MAMBO)}

MAMBO's innovation is the realization that an instrument purpose-built for measuring the $\mathrm{MeV}$ gamma-ray EGB from a $6 U$ CubeSat observatory can provide measurements that are superior to the best currently available in the important MeV energy band. There are two key reasons for this: (1) the EGB is so bright at $\mathrm{MeV}$ energies that it only requires an instrument with a small effective area and (2) the best existing measurements are compromised by the locally generated instrumental background, which scales with the mass of the observatory (figure 2). When designing for MeV EGB measurements, small is beautiful.

The MAMBO mission is designed to employ the standard 6U CubeSat format. CubeSat specifications employ a standard form factor that is scalable in discrete units of $1 \mathrm{U}$, where $1 \mathrm{U}$ is a cube of $10 \mathrm{~cm} \times 10 \mathrm{~cm} \times 10 \mathrm{~cm}$. This standardization of CubeSat design has allowed the deployment of standard launchers that largely decouple CubeSat development from the specifics of the launch vehicle. And the widespread adoption of CubeSat standard launchers by NASA, DoD, and industry is now providing rapid, inexpensive, access to earth orbit. The challenge for MAMBO is the design of a compact spectrometer and spacecraft sub-systems that can meet the limited Space,

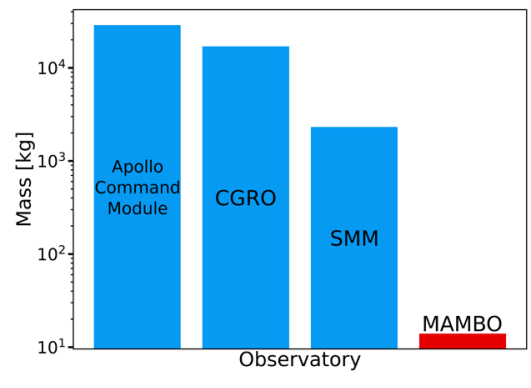

Figure 2. The instrumental effective areas for $M A M B O$ and all previous $M e V E G B$ measuring instruments are within a factor of a few. But the locally generated instrumental background scales with observatory mass. MAMBO will provide higher signal-to-noise measurements because the total observatory mass is two orders of magnitude smaller. 
Weight and Power (SWaP) imposed by the CubeSat format.

3.1 The МАМВО Gamma-Ray Spectrometer. Four top-level requirements drove the design of the gamma-ray spectrometer:

- The spectrometer must cover the $\sim 300 \mathrm{keV}$ to $\sim 30 \mathrm{MeV}$ energy band and have an effective area of $\sim 30 \mathrm{~cm}^{2}$ at $1 \mathrm{MeV}$ (comparable to COMPTEL on CGRO) so that it has the sensitivity to measure the MeV EGB.

- The spectrometer must be compact and efficient so that it can fit the limited Size, Weight and Power (SWaP) budget available in the 6U CubeSat format.

- The spectrometer must be designed to minimize the systematic uncertainties generated by internal instrumental background.

- The spectrometer should have a directional response that drops by about a factor of 5 at $1 \mathrm{MeV}$ for incidence angles beyond $50^{\circ}$ to suppress confusion from off-axis sources.

The MAMBO low-background spectrometer design employs two closely spaced 3"x3" BGO gamma-ray scintillation detectors in an active anti-coincidence well (see figure 3 ). The sides of the anti-coincidence well are constructed from a $1.5 \mathrm{~cm}$ thick annulus of BGO with an outer diameter of $10 \mathrm{~cm}$ and a length of $20 \mathrm{~cm}$. To provide full $4 \pi$ anti-coincidence protection, the well is capped at the bottom with a $1.5 \mathrm{~cm}$-thick BGO scintillator and at the entrance aperture with a $0.5 \mathrm{~cm}$-thick plastic scintillator. This active well arrangement suppresses the energetic particle backgrounds and provides Compton suppression that improves the spectral fidelity.

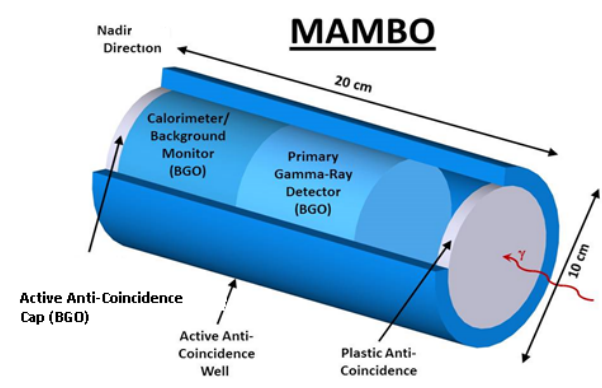

Figure 3. A cut-away drawing of the scintillator layout in the MAMBO spectrometer.

To closely nest the two BGO detectors and meet the low SWaP budget imposed by CubeSat deployment, all of scintillators in the MAMBO spectrometer are read out by silicon photomultipliers (SiPM). The SiPM offers a small, robust, and lowvoltage light sensor that is insensitive to magnetic fields and features gain, response time, and detection efficiency equivalent or superior to traditional photomultiplier tubes (PMTs). Stable operation in a near-space environment, including real-time compensation for temperature-related gain variations, has been demonstrated on a NASA balloon flight [16], and SiPMs are currently being integrated into the upcoming BurstCube CubeSat mission [17]. An appropriate SiPM to baseline for MAMBO is the MicroFJ-60035-TSV from SensL Technologies, Ltd. This device, which only requires $\sim 30 \mathrm{~V}$ bias voltage, also features an effective quantum efficiency of $\sim 40 \%$ at the peak BGO emission wavelength of $480 \mathrm{~nm}$; superior to a PMT. An array of these $6 \mathrm{~mm} \times 6 \mathrm{~mm} \mathrm{SiPMs}$ will be assembled on a custom PCB to match the area of the BGO detectors that is easily accommodated within the small gap $(\sim 3 \mathrm{~mm})$ between the detectors.

MAMBO will employ a new approach for instrumental background suppression. Since the two BGO detectors are identical and forced to a common gain, we can use the back detector (which has its view of the entrance aperture blocked by the front detector) as a time-real monitor of the internal instrumental background. Subtraction of temporally coincident back detector 
(Det2) spectra from the front detector (Det1) spectra helps remove the instrumental background component even during fast variations like those generated by internal radioactivity after passage through the South Atlantic Anomaly. It also suppresses the response to incident gamma-ray flux from confusing off-axis sources that are outside primary entrance aperture. To enable this approach, the front $\mathrm{BGO}$ detector and back BGO detector are automatically controlled by an electronic servo-loop that continuously forces them to a common gain. This real-time gain control scheme employs coincidence of a ${ }^{60} \mathrm{Co}$ calibration photon in one of the BGO detectors with a gate pulse generated by prompt ${ }^{60} \mathrm{Co} \beta$ - decay in a plastic calibration button [18] sandwiched between the detectors.

To quantify this new approach and derive the effective area and angular response as a function of energy, we conducted simulations of the MAMBO spectrometer design using validated GEANT4-based code developed at LANL for modeling the Gamma-Ray Burst Monitor on Fermi. Figure 4 shows the derived energy dependence and effective area of the front and back BGO detectors as a function of photon arrival direction. The spectrometer achieves an effective area at $1 \mathrm{MeV}$ of $30 \mathrm{~cm}^{2}$ for on-axis photons entering the front aperture. Gamma-ray leakage through the back hemisphere is small as represented by the effective area at $1 \mathrm{MeV}$ of $<1 \mathrm{~cm}^{2}$ for most of the solid angle. By subtracting the back detector effective area (Det2) from the front detector (Det1) as a function of incidence angle, one can see how the differencing mode sharpens the angular response and drops the effective area by a factor of six at $1 \mathrm{MeV}$ for incidence angles greater than 50 degrees. MAMBO will therefore have sufficient area to measure the MeV EGB and will be less susceptible to systematics that compromise the best existing measurements.

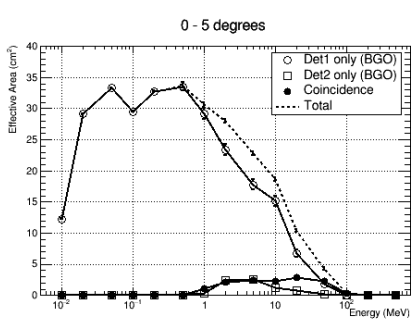

$70-75$ degrees

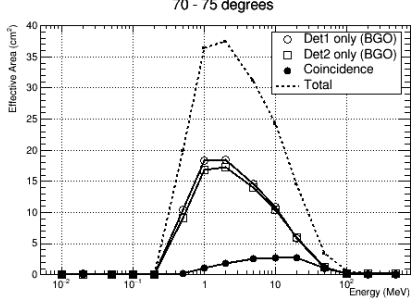

$130-135$ degrees

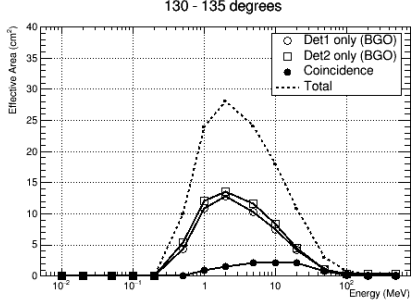

25 - 30 degrees

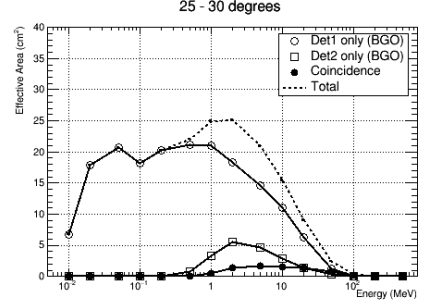

90 - 95 degrees

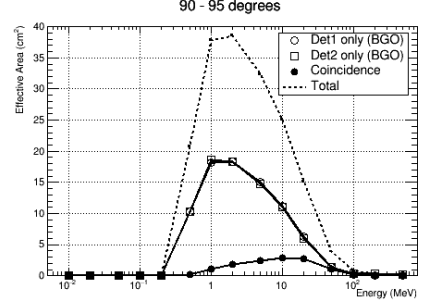

$150-155$ degrees

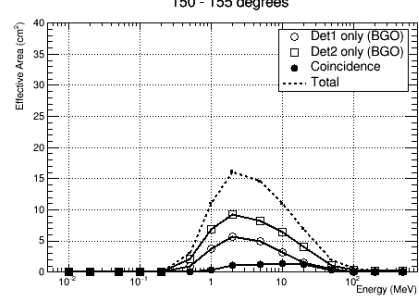

50 - 55 degrees

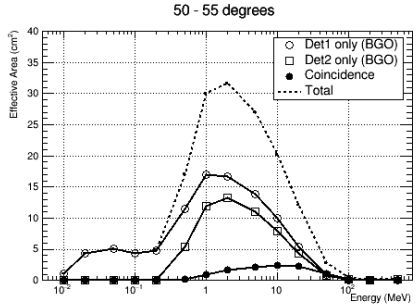

$110-115$ degrees

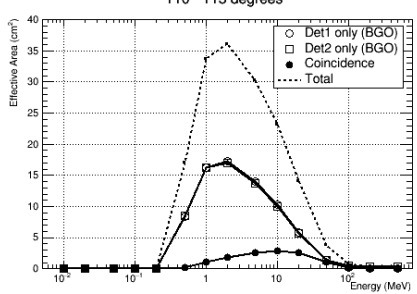

175 - 180 degrees

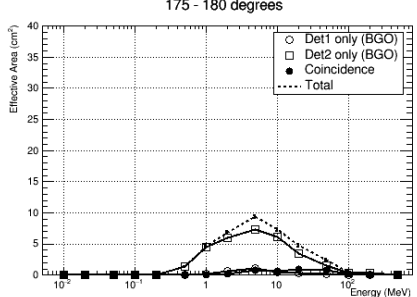

Figure 4. The effective area as a function of gamma-ray energy and incidence angle for the two BGO detectors. The dotted line shows the total effective area when the detector responses are summed. To determine the total effective area in difference mode, one needs to subtract the Det 2 effective area from the Det1 effective area. 


\subsection{The MAMBO CubeSat Bus}

The MAMBO instrument will be deployed in a $6 \mathrm{U}$-class CubeSat that is subdivided into a $3 \mathrm{U}$ instrument compartment and $3 \mathrm{U}$ spacecraft subsystems compartment. The spacecraft subsystems leverage components with flight heritage developed by LANL's CubeSat program that, to date, has launched 15 CubeSats for Agile Space applications.

MAMBO's 6U CubeSat bus will employ a distributed computing architecture with a realtime operating system. There is no central processor that controls the entire system, instead, each node operates independently on an internal network. The nodes in the architecture include the Command and Data Handler, Radio \#1, Radio \#2, the Attitude Determination and Control System, the Power Control System, and the Payload Interface. A block diagram of the architecture is shown in figure 5. Each digital board has a similar layout (template processor, supervisor FPGA, etc.), includes some board specific circuitry, is populated in accordance with that board's specific processing requirements, and plugs into a backplane which provides power and interconnectivity.

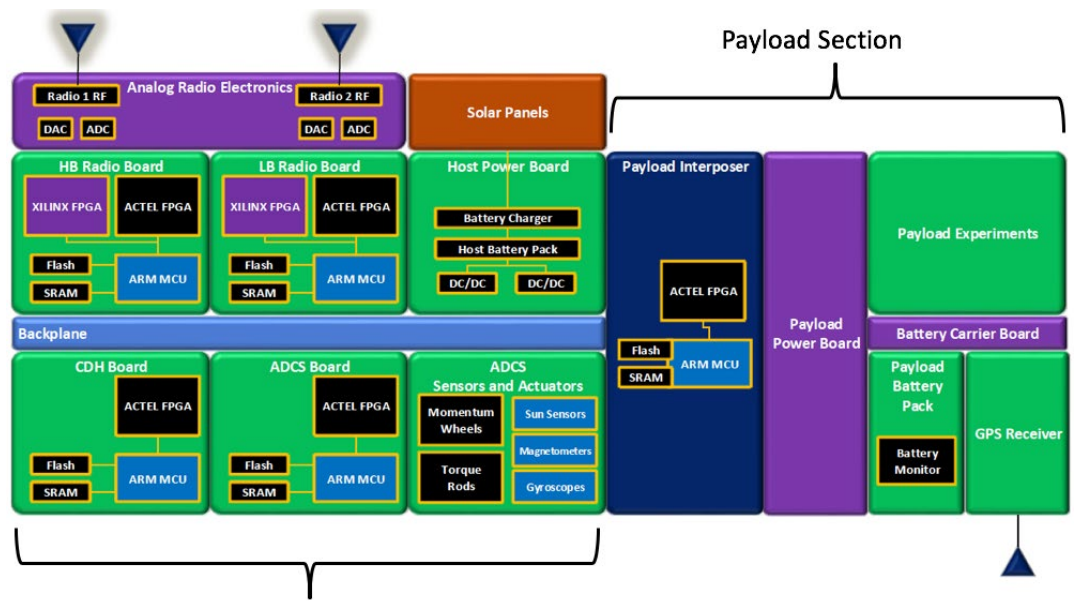

Figure 5. A functional layout of subsystems housed in the $3 U$ spacecraft compartment.
The Command and Data Handler $(\mathrm{CDH})$ is a key spacecraft subsystem. It receives most ground station commands and acts upon them. It also controls power distribution to the other boards and acts as the central communication hub for the internal Spacewire network. The board itself employs an

ARM Cortex-M7 processor running at $216 \mathrm{MHz}$ with $2 \mathrm{~GB}$ of external Flash and also carries an Actel Pro ASIC3 1000 FPGA to assist the on-board processing.

MAMBO will carry two radios on the satellite bus; a high-band radio operating near 2.2 $\mathrm{GHz}$ and a low-band radio operating near $240 \mathrm{MHz}$. Both digital radio boards use the same processor as the $\mathrm{CDH}$ board. The high-band radio uses a deployable helical antenna while the lowband radio uses deployable whip antennas. Data rates using the high-band radio can approach 5 Mbps.

The Attitude Determination and Control System (ADCS) employs three pinhole sun-sensors, two magnetometers, and two MEMS gyroscopes for attitude determination. Attitude actuation is accomplished using four momentum wheels in a pyramid configuration or three magnetic torque rods. Our current system with space heritage [19] achieves a pointing accuracy of about 5 degrees and an attitude knowledge accuracy of $\sim 1$ degree; sufficient for coarse spatial mapping with MAMBO. But we plan to add an on-board star tracking sensor that would enable 1 degree pointing accuracy and attitude knowledge accuracy approaching 1 arcminute. We have developed a star tracker prototype that achieves this accuracy, but it has yet to be demonstrated in flight. 


\section{The Mission Concept}

Our Mission goal is to map the full sky at $\sim 300 \mathrm{keV}$ to $\sim 30 \mathrm{MeV}$ energies during a nominal two year period of operation. Each pointing will cover a field-of-view of $\sim 1$ steradian, and we will use approximately 50 different field centers to help mitigate point source confusion issues. The density of field center concentration will also be biased toward the north and south galactic poles to optimize the collection of MeV EGB measurements and minimize the diffuse contribution from the galactic plane. Using 50 fields will yield $\sim 10^{6}$ second dwell for each field, which is comparable to the nominal 2-week field pointing employed by CGRO.

An important aspect of the mission concept that is still under development is the preferred orbit for MAMBO. The concepts for instrumental background reduction are designed so that MAMBO can take advantage of any launch opportunity. But equatorial LEO orbits that avoid SAA passes are an attractive option because they minimize long-term activation that typically becomes the main source of instrumental background at high inclination $\left(\mathrm{i}>10^{\circ}\right)$ [15].

Another attractive mission concept would be to deploy MAMBO in a lunar orbit and employ the Lunar Occultation Techniques [20] being developed for the Lunar Occultation Explorer. The lunar background environment is slowly varying and the LOT temporal modulation will provide an additional tool for removing confusion generated by strong $\mathrm{MeV}$ emitting discrete sources. The disadvantage of this approach is that there are fewer launch opportunities for lunar orbit insertion and it requires significantly greater capability in the communications architecture than we are currently baselining for MAMBO.

The baseline communication architecture for MAMBO leverages the ground segment technology developed by LANL's CubeSat program. The simple, low-cost ground station will be a replica of the existing LANL ground stations. The user interface software will be adapted for MAMBO's requirements, but it already contains the necessary features for establishing communications, uploading new parameters, and downlinking data files. We plan to use the LANL Satellite Operations Center as the primary hub of operations, but remote access to the ground station computers to command the satellites is also possible. Automated downlink of available data files is already supported in the existing architecture and only minimal commanding of MAMBO will be needed, so operations will require a low level of effort.

\subsection{Summary}

While not a general solution for exploring the Universe in the important $\mathrm{MeV}$ band, CubeSats can provide a useful platform for the study the MeV EGB light. We presented a design for a gamma-ray spectrometer capable of both providing quality measurements of the spectrum and angular distribution of the MeV EGB and meeting the challenges associated with the tight Size, Weight, and Power (SWaP) constraints imposed by the goal of deployment on a $6 \mathrm{U}$ CubeSat. We also presented a mission concept called MAMBO that, during a nominal two year baseline mission, would be able to collect measurements of the MeV EGB background that are superior to the best currently available and place important constraints on MeV EGB origin.

\subsection{Acknowledgement}

Research presented in this paper was supported by the Laboratory Directed Research and Development program of Los Alamos National Laboratory. 


\section{References}

[1] V. Schonfelder et al., The First COMPTEL source catalogue, Astronomy and Astrophysics Supplement, vol.143, Number 2,(2000)

[2] W.T. Vestrand et al. The Solar Maximum Mission Atlas of Gamma-Ray Flares, Ap. J. Supp.Ser. Volume 120, pp. 407-467, (1999)

[3] A. W. Strong et al., Diffuse galactic hard X-ray and low-energy gamma-ray continuum, Astronomy and Astrophysics Supplement, v.120, p.381-387, (1996)

[4] M. Ajello et al., The MeV Background, Astro2020: Decadal Survey on Astronomy and Astrophysics, science white papers, no. 290; Bull. American Astronomical Society, Vol. 51, Issue 3, id. 290 (2019)

[5] J. I. Trombka et al., Reanalysis of the Apollo cosmic gamma-ray spectrum in the 0.3 to $10 \mathrm{MeV}$ energy region, Astrophysical Journal, 212, pp. 925-935 , (1977)

[6] G. Weidenspointner et al., The cosmic diffuse gamma-ray background measured with COMPTEL, AIP Conference Proceedings 510, p. 467, (2000); https://doi.org/10.1063/1.1307028

[7] S. C. Kappadath et al., The preliminary cosmic diffuse $\gamma$-ray spectrum from $800 \mathrm{keV}$ to $30 \mathrm{MeV}$ measured with COMPTEL, Astronomy and Astrophysics Supplement, v.120, p. 619-622 , (1996)

[8] K. Watanabe et al., The MeV cosmic gamma-ray background measured with SMM, AIP Conference Proceedings 510, p. 471, (2000); https://doi.org/10.1063/1.1303252

[9] M. Ackermann et al., The Spectrum of Isotropic Diffuse Gamma-Ray Emission between $100 \mathrm{MeV}$ and $820 \mathrm{GeV}$, Astrophysical Journal, 799, 86, (2015)

[10] K. Ahn and E. Komatsu, Dark matter annihilation: the origin of cosmic gamma-ray background at 1-20 MeV,Phys. Rev. D, 72,1 (2005)

[11] P. Ruiz-Lapuente et al., The origin of the Cosmic Gamma-ray Background in the MeV Range, Astrophysical Journal, 820, (2016)

[12] M. Ajello et al. The Evolution of Swift/BAT Blazars and the Origin of the MeV Background, Astrophysical Journal, 699, pp. 603-625 (2009)

[13] Y. Inoue et al. The Cosmic MeV Gamma-Ray Background and Hard X-Ray Spectra of Active Galactic Nuclei: Implications for the Origin of Hot AGN Coronae ,Astrophysical Journal, 672, L5, (2008)

[14] B. Lacki et al., The Star Forming Galaxy Contribution to the Cosmic MeV and GeV Gamma-Ray Background, Astrophysical Journal, 786, 1 (2014)

[15] P. Cumani et al., Background for a gamma-ray satellite on a low-Earth orbit, Experimental Astronomy, https://doi.org/10.1007/s10686-019-09624-0, (2019)

[16] P. Bloser, et al., The Advanced Scintillator Compton Telescope (ASCOT), Jour. It. Astro. Soc., (2019)

[17] J. Racusin, et al., BurstCube: A CubeSat for Gravitational Wave Counterparts, Proceedings of Science (ICRC2017), 760 (2017)

[18] D. Forrest et al., An electronically Gated Gamma and X-Ray Calibration Scheme, Nuc. Instruments and Methods, 101, pp. 567-572. (1972)

[19] J.A. Wren et al., The third-Generation Los Alamos Designed 1.5U CubeSat Attitude Determination and Control System, $31^{\text {st }}$ Annual AIAA/USU Conf. on Small Satellites, SSC17-VII-07 (2017).

[20] R.S. Miller and D. J. Lawrence, First Light: MeV Astrophysics from the Moon, Astrophysical Journal, 823, L31 (2016) 